\title{
Espacios públicos y dimensión informal de la vida urbana en Quito
}

\section{Public spaces and informal dimension of urban life in Quito}

\author{
${ }^{1}$ Prof. Arq. Alessio Pea, Ph. D., \\ ${ }^{2}$ Prof. Arq. Matteo Clemente, Ph. D. \\ ${ }_{1}$ Prof. Arq. del Observatorio Urbano y del Paisaje, Facultad de Arquitectura y Urbanismo, \\ de la Universidad Tecnológica Equinoccial. Quito-Ecuador. \\ 〈alessio.pea@ute.edu.ec〉, ‘alessiopeadesign@gmail.com〉.
}

${ }^{2}$ Prof. Arq. del Departamento de Arquitectura y Proyecto, Facultad de Arquitectura, de la Sapienza Universidad de Roma. Roma, Italia. 〈matteo.clemente@uniroma1.it〉,〈clementematteo@yahoo.it».

Recepción / Received: 30, 10, 2017.

Aceptación / Accepted: 30, 11, 2017.

Publicado / Published: 15, 01, 2018.

Resumen: El presente estudio analiza los espacios públicos urbanos de la ciudad de Quito, desde una lectura que relaciona las características de los lugares, con las dinámicas de uso y la percepción individual y colectiva de los usuarios. En primer lugar ha sido posible estipular un cuadro taxonómico de referencia, mediante un atento trabajo sistemático de los espacios urbanos analizados. Se deduce un uso informal de los parques públicos por parte de los habitantes durante los días de fiesta, para disfrutar al aire libre. Lo que parece muy relevante es como, en ausencia de fuertes contenidos formales, los espacios destinados al área verde con algún arbolado, se transformen en espacios aceptados donde la comunidad local interacciona y cumple con una actividad de esparcimiento. La ciudad informal se auto-organiza con usos espontáneos de los espacios, siguiendo instintos y respondiendo a necesidades contingentes de manera adaptativa.

Palabras clave: actividades humanas, espacio público, dimensión informal, paisaje sensible, uso común

Abstract: The present study examines the urban public spaces in Quito, through a reading key that connects the characteristics of the places, with the dynamics of use and the individual and collective perception of the users. Through the systematic mapping of urban space with cards, a taxonomic reference framework was built. You can observe an informal use of the public parks, which are populated by the inhabitants on holiday days, to spend time in the open air. What appears very remarkable is that, in absence of strong formal contents, simple surfaces used on a lawn with few trees, they become living spaces where local community interacts and performs activities. The informal city self-organizes through the spontaneous use of spaces, following instances from the bottom and responding to contingent needs in an adaptive manner. If the creation of consumer-oriented public spaces and specialized and segregated mono-functional structures have created a new geography of social exclusion in the western city, Quito's public spaces (which is a poor metropolis case) characterized by unpredictable and spontaneous uses, are socialization spaces where it is possible to cultivate a true relationships with the community. 
The study of informal practices can be a useful reference in Western metropolises, for the implementation of urban tactics and bottom-up regeneration practices, taking into account the everyday life of ordinary spaces.

Keywords: Common Ground, Human Activities, Informal dimension, Sensitive landscape, Public space

\section{INTRODUCCIÓN}

El modo de leer, interpretar y planear las ciudades en la era de la globalización, es homologado y homogéneo en el contexto internacional. El modelo de referencia es el funcionalista a la ciudad occidental, organizada a partir de un sistema jerárquico de control de los espacios, conocido hoy como idea de eficiencia y calidad de los servicios en términos de smartcity. Este modelo, a partir del cual se cumplen valoraciones comparativas entre la ciudad, utiliza parámetros y normas que no son aplicables en todas las situaciones. El riesgo es proponer soluciones de proyectos no coherentes con el contexto urbano y estrategias de planificación que no toman en cuenta las realidades socioeconómicas.

El tema del espacio público se ha convertido en una cuestión fundamental en las smartcity occidentales, tambien en los lugares más pobres del planeta, que no por esto pueden definirse como menos “inteligentes". En particular, en América del Sur, en ciudades como Río de Janeiro, en el
Brasil; Bogotá o Medellín, en Colombia, se ha visto en los últimos años que se han emprendido muchas iniciativas y proyectos sobre el espacio público de áreas urbanas marginales y con mayor malestar social, promoviendo las aspiraciones de las comunidades locales por una sociedad más pacífica y productiva. Con la creación de espacios públicos dentro de los contextos urbanos a menudo se da la recualificación arquitectónica y social, con una estrecha correlación entre calidad de los espacios urbanos, en términos de interacción social y decadencia de las situaciones de conflicto social y la criminalidad.

Cuánto se tiene que hacer con las metrópolis de Sudamérica o con otras realidades urbanas extra-europeas, la idea de espacio público tiene connotaciones diferentes, incluso el estilo de vida de los habitantes y el contexto socioeconómico que lo determina es diferente. Resulta necesario por lo tanto valerse de algunos criterios estimativos obtenidos de una cultura eurocéntrica, para encajar en el tema del espacio público a la luz de los usos y la 
cultura local, y de la vida que se desarrolla en los espacios urbanos abiertos en lugares del planeta menos occidentalizados.

Aunque en el mundo occidental globalizado el parque urbano, con toda su implicación atada al bienestar individual y colectivo, asumió el papel social de la plaza histórica, el espacio público de las ciudades europeas todavía está atado a la idea de la representación del orden político y social. También en sus decrecimientos de los parques: urbano y lineal, infraestructura verde, waterfront urbano, el espacio público de la ciudad occidental es un lugar muy formalizado que se favorece a menudo de márgenes arquitectónicos calificados o de un dibujo firmado por famosos arquitectos que escribe, y a veces prescribe, los usos sociales.

Al contrario, el espacio público de las áreas urbanas en las ciudades de Sudamérica, como ocurre en general en los lugares más pobres del mundo, tiene un uso particularmente informal que está estrechamente relacionado con la voluntad de estar al aire libre.

El presente estudio toma en consideración los espacios públicos urbanos de Quito en Ecuador. Con las debidas diferencias, ya que cada ciudad es diferente de la otra por definición, la ciudad de Quito puede ser representativa, por algunos aspectos donde se unen el uso informal del espacio público y las dinámicas sociales, de América del Sur. En particular se han examinado algunos de los espacios urbanos abiertos de Quito, con sus características morfológicas y funcionales, ya que son percibidos, utilizados y aceptados de manera informal por parte de los ciudadanos.

Quito es la capital del Ecuador, se encuentra a 2.763 msnm, su población, según el Censo de Población y Vivienda de 2010, se encuentra en 2’231.705 habitantes, ${ }^{1}$ con una extensión territorial de $290 \mathrm{~km}^{2}$.

Gracias a las políticas de gestión del territorio de la última administración pública, la ciudad vive un proceso de renovación urbana, vinculada también a la reactivación económica. Existe una política estable, resultado de la permanencia del gobierno de izquierda, que se mantiene desde hace 15 años, este ha impulsado la realización de numerosas inversiones y obras sociales $\mathrm{y}$ culturales, parques, plazas y jardines e importantes infraestructuras como la autopista avenida Simón Bolívar que conecta la ciudad de Quito con el aeropuerto Internacional Mariscal Sucre, localizado en el sector de Tababela. Actualmente está en

\footnotetext{
${ }^{1}$ http://datos.quito.gob.ec/dashboards/10/demografia〉.
} 
fase de construcción el primer metro subterráneo, además, la ciudad cuenta con un sistema bastante eficiente de rental bike (BiciQ), con una red de infraestructuras para la reposición de las bicicletas, situado en las principales plazas de la ciudad y en los barrios más concurridos.

A nivel social, existe todavía un gran malestar, sobre todo, al interior de las familias individuales, esto debido a que las condiciones de vida son muy inestables, lo que genera una gran incertidumbre acerca del futuro económico; se aprecia una alta tasa de suicidios entre los más jóvenes, 15-35 años, lo que representa para las jóvenes generaciones un declive social, sin hablar de otras situaciones más graves, que desembocan en abusos sexuales familiares.

A pesar de este escenario social, la primera impresión que se tiene al visitar la ciudad de Quito, en especial en los días de fiesta, es que los ciudadanos tienen preferencia por el descanso en los parques urbanos.

Quito resulta ser un laboratorio importante para estudiar las dinámicas de uso del espacio urbano abierto, no solo en la perspectiva de definir un cuadro de referencia para la planificación urbana y la previsión de nuevos escenarios de intervención, sino también para proveer opciones de análisis desde realidades occidentales más atadas al dibujo formal de la ciudad. Hay mucho que aprender de los usos espontáneos de los lugares en términos de valoración de las preferencias individuales y colectivas, de concurrencia y uso, de percepción del bienestar o malestar.

El presente estudio discute sobre el tema de los espacios urbanos abiertos de la ciudad de Quito, a partir de la percepción de los habitantes y de las dinámicas de uso espontáneo e informal. Pone en relación las calidades percibidas por los habitantes y el nivel de interacción social con las características arquitectónicas de los lugares analizados, se pueden obtener reflexiones útiles para realizar un adecuado planeamiento de los espacios urbanos abiertos. Quito con sus usos informales puede ser un laboratorio de análisis muy provechoso para definir líneas que conducirán las intervenciones en el espacio público de la ciudad contemporánea.

La investigación sobre los espacios públicos de la ciudad de Quito, parte de una actividad más amplia realizada en el ámbito de la Facultad de Arquitectura y Urbanismo de la Universidad Tecnológica Equinoccial (UTE), adquiere, por lo tanto, relevancia no solo en el contexto urbano, sino también en el panorama internacional. 


\section{METODOLOGÍA Y MATERIALES}

Quito posee un área urbana con elevada concentración de viviendas, se extiende de norte a sur a lo largo de $50 \mathrm{~km}$ y de este a oeste en aproximadamente unos $10 \mathrm{~km}$. Para realizar nuestra investigación se ha seleccionado como área de estudio la parte central de la ciudad, que incluye el casco antiguo, el centro histórico, político $\mathrm{y}$ administrativo de la ciudad y las áreas donde hay la mayor concentración de espacios públicos. El área posee una superficie de 50 $\mathrm{km}^{2}$ y una dimensión de $8 \mathrm{~km}$ por $6,5 \mathrm{~km}$ (figura 1).

La investigación se basó en una actividad coordinada de laboratorio, donde 36 estudiantes de la Facultad de Arquitectura y Urbanismo de la UTE, subdivididos en seis grupos de trabajo y distribuidos en los seis cuadrantes localizados, desarrollaron el análisis de los espacios públicos existentes en el área urbana seleccionada (figura 1).

Cada grupo de trabajo, compuesto por 6 estudiantes, ha desarrollado investigaciones directas en el área asignada, sobre la base de una metodología común que ha previsto objetivamente la recopilación de fichas que contienen datos cuantitativos medibles, $\mathrm{y}$ cualitativos, deducibles de la lectura de los datos y de las entrevistas in situ.

El trabajo de análisis e investigación sobre el campo ha sido desarrollado de forma dinámica basado en la observación temporal y directa, durante un período situado entre los meses de abril y julio de 2017.

La ficha aplicada a cada lugar fue programada de manera que cada espacio público estudiado y analizado fuera catalogado, indicando el año de construcción/regeneración, el sector o barrio de pertenencia y la dimensión en $\mathrm{m}^{2}$.

Paralelamente a estos datos ha sido útil identificar cada espacio público por su tipología, así: 1. parque lineal, 2. parque urbano, 3. plaza, 4. bosque urbano, 5. jardín, mirador, 6. espacio para fitness, 7. espacio lúdico y recorridos peatonales. Además se localizó la función correspondiente a cada espacio público: cultural, deportista, educativa, lúdica, natural, recreativo, social, comercial y tiempo libre.

En particular han sido localizadas y clasificadas en la ficha las características funcionales y los elementos presentes en cada espacio público según la siguiente lista de referencia: 1. encuentro, 2. descanso, 3. baile, 4. educación, 5. gastronomía, 6 . comerciantes ilegales, 7. eventos, 8. teatro, 9. música, 10. área para perros, 11. granjas 
urbanas, 12. huertos urbanos, 13. jardín, 14. arquitectónicas, 29. punto de informaciones, protecciones y barreras, 15. iluminación, 16. 30. servicios para los ciudadanos, 31. huertos urbanos, 17. gimnasia, 18. voleibol, almuerzo informal, 32. wi-fi libre, 33. 19. tenis, 20. baloncesto, 21. fútbol, 22. mobiliario urbano, 34. parqueadero, 35. exposición, 23. arte urbana, 24. patio de jardinera, 36. componente del agua, 37. juego, 25. servicio médico móvil, 26. componente de vegetación, y 38. cámaras de laboratorios temporales, 27. conexión con seguridad.

transporte público, 28. barreras 


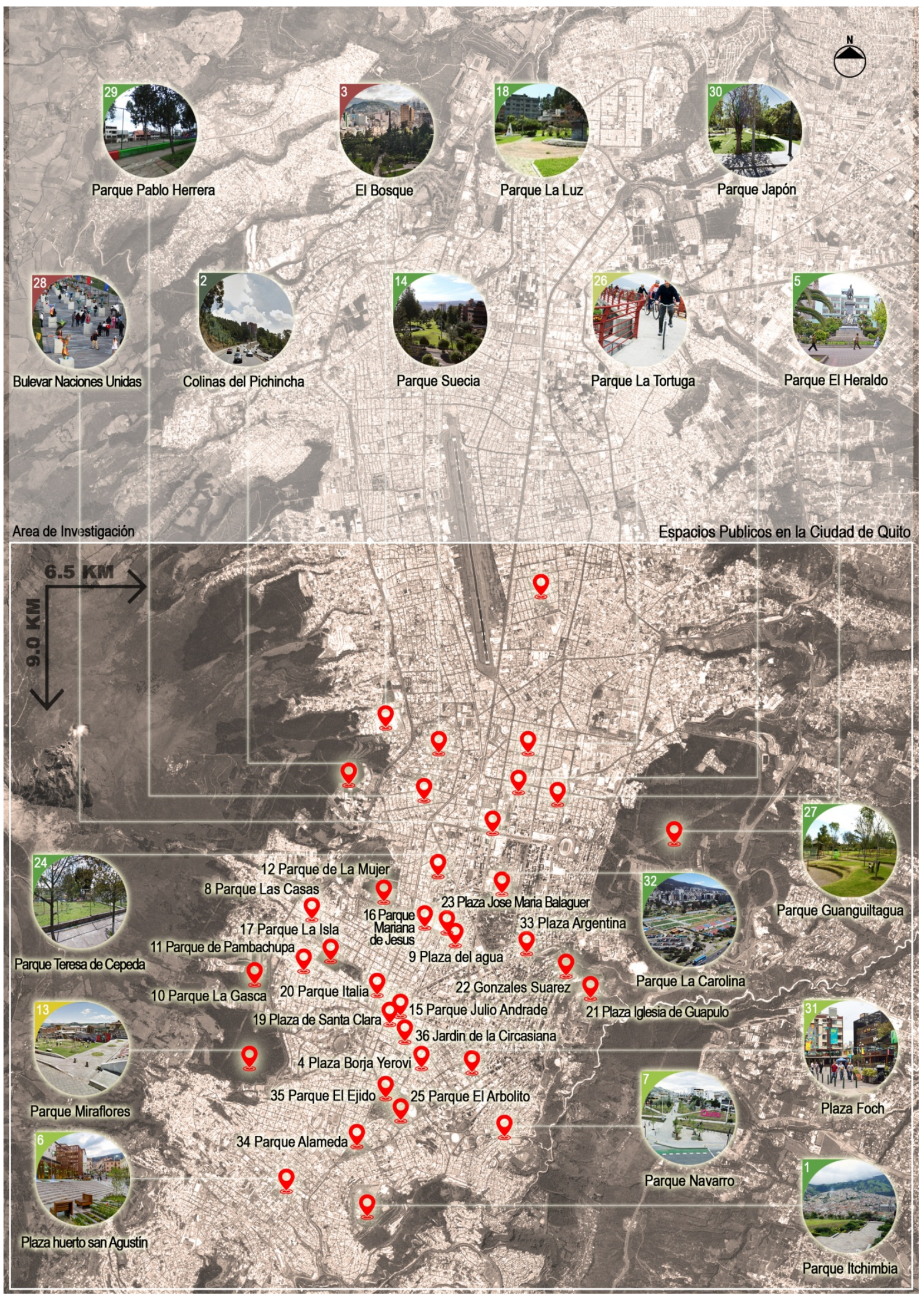

Figura 1. Espacios Públicos investigados. Elaboración: Federica Perissi, 2017. 
Por la observación directa y las reelaboraciones gráficas en laboratorio, han sido identificados los usos predominantes, los flujos de las personas y las características peculiares de cada espacio.

Por las entrevistas realizadas a los usuarios del espacio público, se han recopilado datos relativos a las valoraciones subjetivas y a las preferencias individuales, tratando de interpretar un juicio sobre la calidad del espacio urbano, por cómo es percibido, y no limitado sencillamente al paradigma visual (Pea 2015).

En particular, al poner en relación la percepción y el uso de los espacios urbanos de parte de los ciudadanos con las características del lugar, se han hecho valoraciones sintéticas considerando, la capacidad de engendrar división e integración entre los usuarios.

\section{RESULTADOS}

Los datos obtenidos han sido organizados según un cuadro taxonómico, que ha puesto en evidencia las características y usos predominantes de los espacios públicos de Quito. La mayor parte de los espacios son parques públicos, con una reducción de parque urbano, jardines y parques lineales; solo 5 son plazas (figura 6), no hay calles para las compras en el sentido occidental, pero todos los tipos de espacios se han caracterizado por un uso informal y libre de parte de los ciudadanos. De la elaboración de los resultados, que incluyen las valoraciones de los ciudadanos, se observan características positivas y negativas de los espacios públicos a Quito (figura 2).

Emerge una fuerte capacidad de estos lugares de ser reconocidos como lugares de encuentro, en el 97\% de los casos, y como lugares para compartir y el relajamiento (94\%) de las entrevistas. El componente natural casi siempre está presente y la vegetación está bien mantenida por parte de la administración municipal, en el 92\% de los casos estudiados. Los espacios públicos que presentan una calidad y una percepción elevada de parte de los ciudadanos entrevistados resultan ser: Parque La Carolina; Parque El Ejido; Parque Las Casas; Parque del Agua; y Plaza Huerto San Agustín.

Sin embargo, se puede apreciar algunos aspectos negativos y carencias estructurales de algunos espacios; solo el $86 \%$ de estos lugares presentan mobiliario de iluminación que permite la utilización nocturna; y, solo el $39 \%$ de los lugares están dotados de cámaras de seguridad.

Pocos espacios son destinados al parqueadero de autos, ausentes en el $44 \%$ 


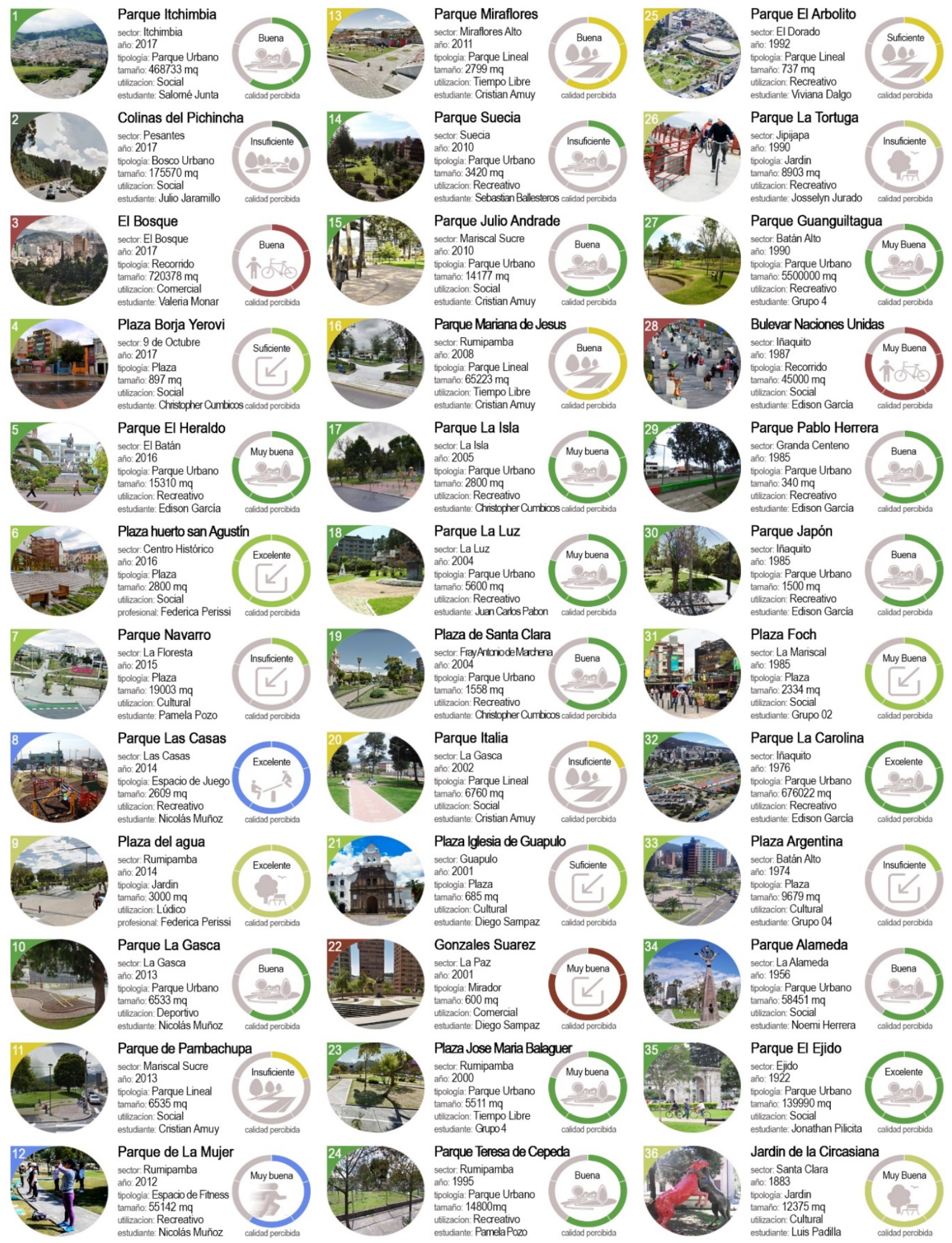

Figura 2. Cuadro sinóptico espacios públicos. Elaboración: Federica Perissi, 2017. 
de los casos, y solo el $33 \%$ de los espacios públicos no poseen barreras perimétricas, como limitación del acceso libre, en la mayor parte de los casos, en todas las horas del día y la noche.

Otro dato relevante y con un fuerte impacto negativo es la falta de condiciones de accesibilidad para las personas minusválidas (44\%). La administración está dotando en el casco antiguo de plazas destinadas como puntos de información (47\%), y de servicios médicos públicos (17\%) para los visitantes, sin embargo, no logran cubrir todavía ni la mitad de todos los espacios públicos presentes.

Tan solo el 28\% de los espacios urbanos cuenta con red de wi-fi municipal para la libre navegación de internet; y menos de la mitad de los espacios públicos tienen piletas, juegos de agua y agua potable (47\%).

Todos estos datos justifican, en parte, la falta de visitantes en los espacios públicos durante la noche, lo que provoca una elevada percepción de inseguridad (Chiesi 2004), sobre todo en horas de la noche.

A partir del análisis efectuado sobre los espacios públicos, las áreas que presentan una carencia de servicios y una menor calidad son:

1. Parque Italia,

2. Parque La Tortuga,
3. Plaza Argentina,

4. Parque Navarro,

5. Parque Suecia, y

6. Parque de Pambachupa.

Se identifican tres casos entre los estudiados, que son calificados como representativos de la ciudad:

1. Parque La Carolina,

2. Parque del Agua, y 3.

3. Plaza Huero San Agustín.

\section{Parque La Carolina}

Se encuentra en el sector de Iñaquito, zona neurálgica de la vida política, financiera $\mathrm{y}$ comercial de la ciudad. Por su forma rectangular, la posición y la relación con lo construido, reviste una gran importancia sociocultural. Presenta una aceptación muy elevada durante el fin de semana y durante los días festivos, cuando "ocurren desplazamientos de masa de la población [...] típico, de las áreas urbanas a más elevada concentración” (Alberti 2012).

Según una costumbre sudamericana, los visitantes se ven muy atraídos por lugares de socialización y encuentro en el espacio urbano abierto justo en los fines de semana (Nessi 2010). Luego del análisis sobre las actividades que se desarrollan dentro del espacio público (Gehl 2014), destaca la práctica deportiva. 


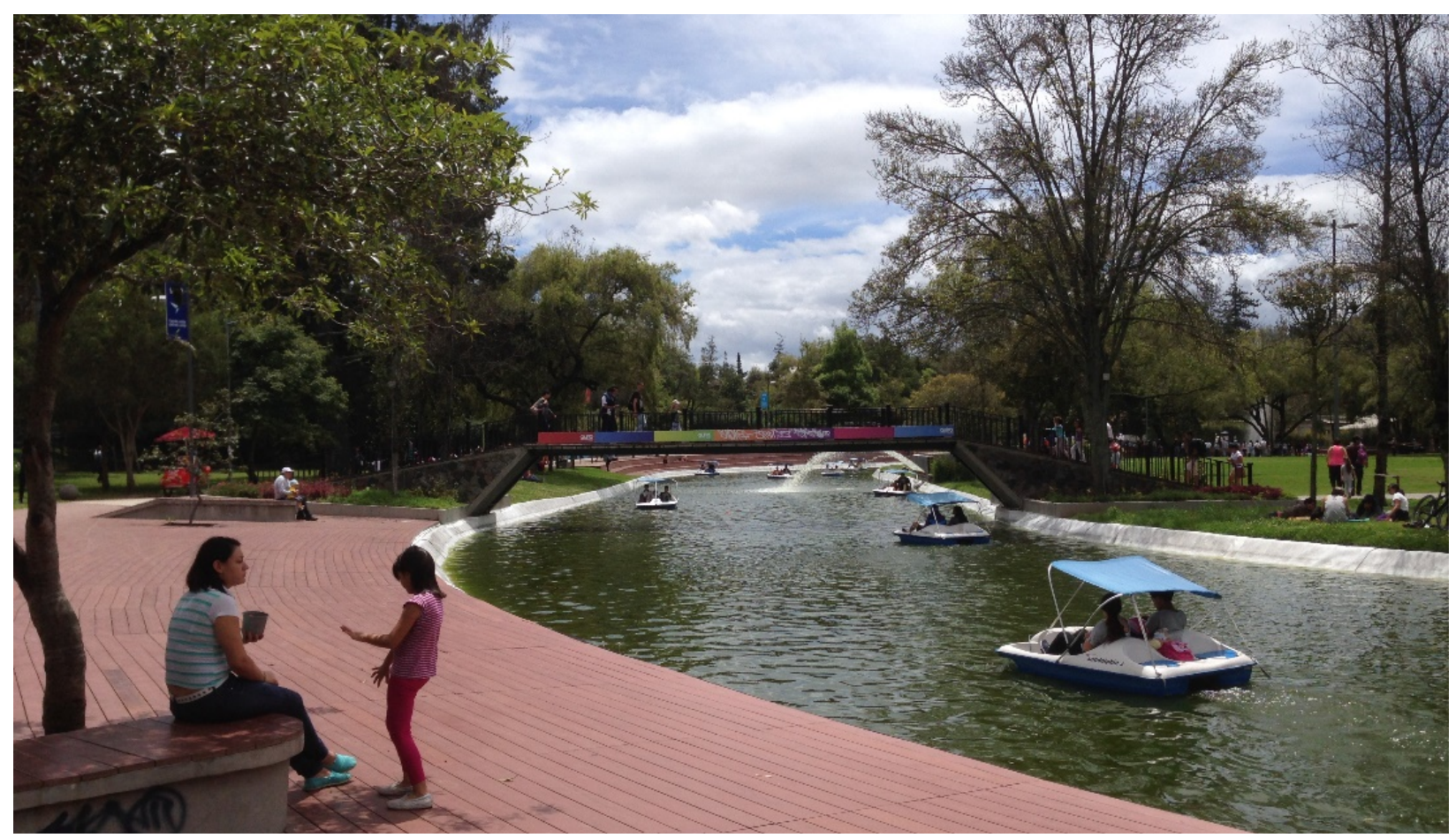

Figura 3. Área de la Laguna, Parque La Carolina. Foto: Alessio Pea, 2017.

La pista de atletismo, se encuentra ubicada al centro del parque y está abierta para el uso de todos los visitantes, además que pueden compartir diferentes aparatos para todo el público.

El parque es abierto, no posee barreras y permite el acceso a cualquier punto a lo largo del perímetro. Al interior están presentes recorridos peatonales que se van interceptando con planos más naturales donde predomina la vegetación (figura 3), según un dibujo articulado, que define una red de espacios urbanos con una fuerte capacidad conectiva (Pea 2016).

Además, las actividades promovidas por la administración municipal dentro del espacio urbano, contribuyen a una regeneración sociocultural continua por la ciudad, favoreciendo los flujos peatonales en los espacios urbanos colindantes (Clemente, 2014).

\section{Parque del Agua}

Está situado cerca al gran Parque La Carolina y puede ser considerado como un parque de encuentro. Este proyecto y su realización estuvieron a cargo de la institución pública que administra los recursos hídricos, el Empresa Pública Metropolitana de Agua Potable y Saneamiento (EPMAPS), de Quito.

Este espacio público se realizó con el fin de sensibilizar a la población hacia un cambio de actitud en cuanto a la disminución 


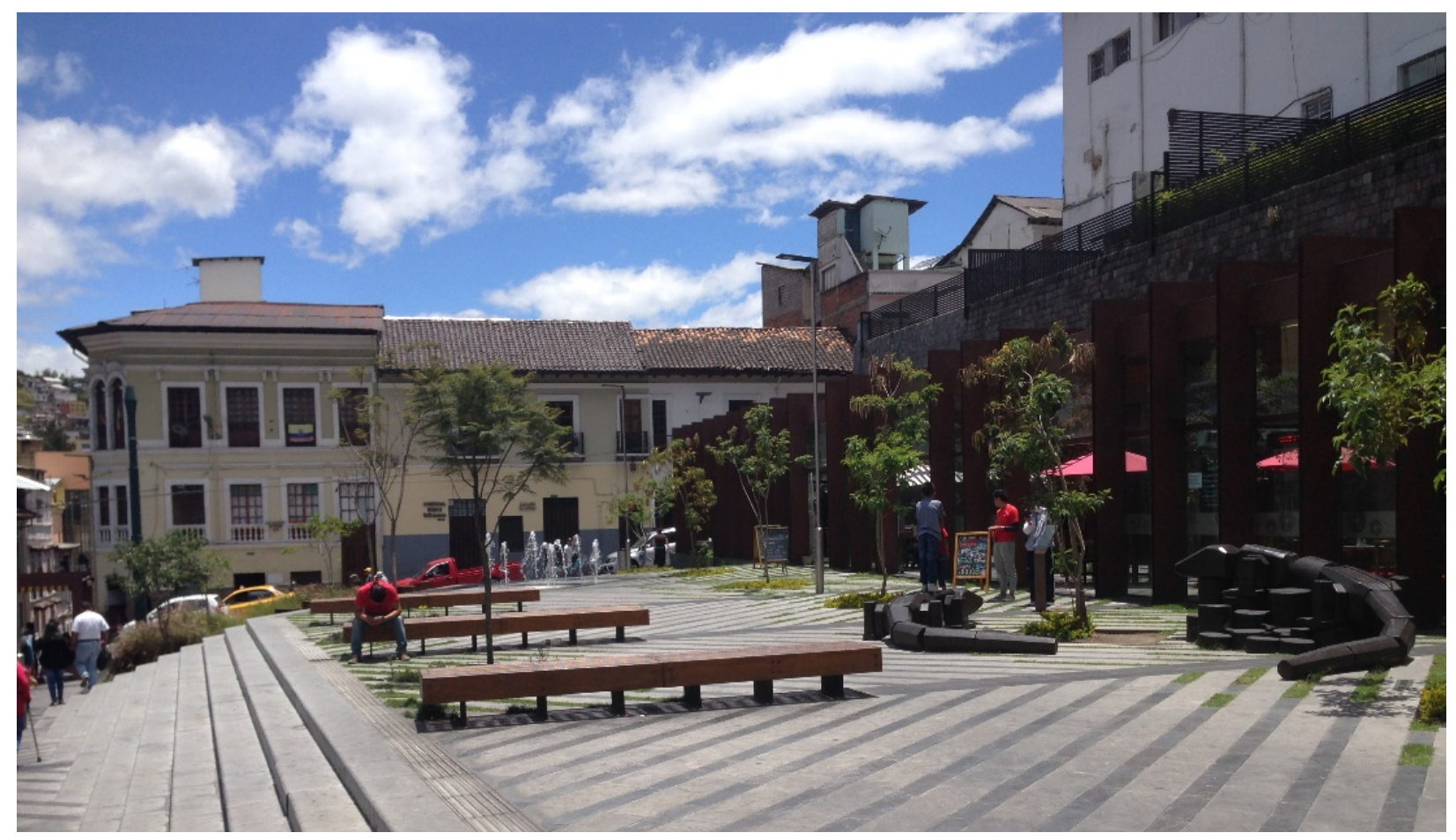

Figura 5. Mobiliario urbano, Plaza Huerto San Agustín. Foto: Alessio Pea, 2017.

del despilfarro en el uso del agua. En su interior están presentes diferentes paneles explicativos que ayudan al usuario a conocer la historia hidráulica de Quito.

El diseño formal del jardín es de tipo céntrico, de hecho todos los recorridos existentes confluyen en la parte central donde está presente la gran fuente de agua circular.

Perimetralmente están dispuestas diferentes mesas y pérgolas que constituyen puntos de parada y encuentro. Además hay juegos con flujos de agua (figura 4), que en los días más calientes siempre son visitados por niños.

A pesar de lo reducido del tamaño del jardín, la cantidad y calidad de flores presente, convierte este espacio público en un lugar único, en el cual se puede disfrutar del entorno y compartir experiencias (Birozzi y Pugliese, 2007).

El nivel de bienestar percibido es muy alto y el jardín presenta calidad en el proyecto y diseño que otros espacios no tienen. Es visible el mantenimiento permanente de las áreas verdes y la promoción de la educación a la vida cívica: "Es muy importante la señalización que motiva a las personas a cuidar este espacio público".

\section{Plaza Huerto San Agustín}

En el año 2016 se completó la trasformación de una plaza localizada en el casco antiguo de la ciudad de Quito -según la UNESCO la mejor guardada de 
Sudamérica- por la demolición de un anónimo centro comercial y así reponer a la ciudad el espacio que perteneció a los huertos urbanos del convento barroco adyacente.

La ubicación de la plaza es de por sí una encrucijada que conectan actualmente polos de interés cultural como el Museo de Arte Colonial, el convento de la Iglesia de La Merced, volviéndose así un centro de atracción enriquecido por una arquitectura contemporánea que se pone en diálogo con el tejido barroco preexistente (figura 5).

Según los análisis efectuados, el proyecto se vuelve un lugar de identidad en el casco antiguo de Quito y reengendra flujos sociales que interesan al casco histórico. Según una relectura de los flujos relativos basados en las actividades humanas obligatorias, voluntarias y sociales (Gehl 2014), es posible atribuir a esta intervención urbana la responsabilidad del paso de la calle Mejía de mera infraestructura vial a escenario potencialmente inagotable de comunicación y cambio (Delgado 2011). La abertura de la calle que se convierte en plaza constituye pues un punto de parada para los flujos intensos que ocurren durante el día. En otras palabras también garantiza una imagen urbana de alta calidad para los habitantes más humildes, respetando el concepto de Borja y
Muxí: "no hay derroche en dar la riqueza a los pobres" (Borja y Muxí, 2000).

\section{DISCUSIÓN Y CONCLUSIONES}

Como resultado del análisis (figura 6) de los casos de estudio, el sitio público en Quito con mayor presencia es el parque urbano por encima de la plaza. Esta es una tendencia en desarrollo en todo el mundo occidental; es evidente que el parque urbano contemporáneo está asumiendo en sí todos los valores formales y funcionales de la plaza.

El parque contemporáneo a menudo es dibujado por artistas y paisajistas que exaltan valores de tipo ecológico, figurativo $\mathrm{y}$ formal, asumiendo con ello la identidad de las señales del proyecto.

Se piensa en las numerosas intervenciones de los WEST8, que han rediseñado paisajes, creando islas verdes artificiales como "Governors Island", en Nueva York-EE.UU., o sistemas lineales como "Madrid Río", en el corazón de Madrid, que se localiza sobre una vasta superficie. El parque contemporáneo se presenta como un sistema fuertemente equipado para acoger y estimular su uso.

El "Millenium Park" de Chicago (19982006), en tal sentido, es un sitio que cumple con una serie de funciones y concentra muchas actividades. Con el auditorio de F. 
Gehry, que hospeda por diez semanas conciertos de música clásica, la fuente interactiva con juegos de agua, crown fountain, el escultura/símbolo de Anish Kappor, los Lurie Garden de Kathryn Gustafson, Piet Oudolf y Robert Israel, este parque se ha convertido en una de las atracciones más populares de la ciudad de Illinois. También en sus decrecimientos más modestos, que no llevan el nombre de artistas internacionales, los parques urbanos de pequeñas ciudades acogen muchas instancias $y$ tienen que satisfacer numerosas expectativas políticas y económicas, más allá de las sociales, apuntando a la construcción de lugares estéticos que rescaten la imagen urbana en un momento en que las ciudades compiten entre ellas para atraer visitantes, ciudadanos, turistas e inversionistas.

Los parques de Quito son bastante modestos en sus contenidos formales, no llevan nombres de artistas y no tienen logo para atraer flujos económicos y de consumo. La comparación con el modelo de smartcity occidental es provechosa sobre este plan de análisis y su contribución a la investigación, es considerar contextos socioeconómicos muy diferentes.

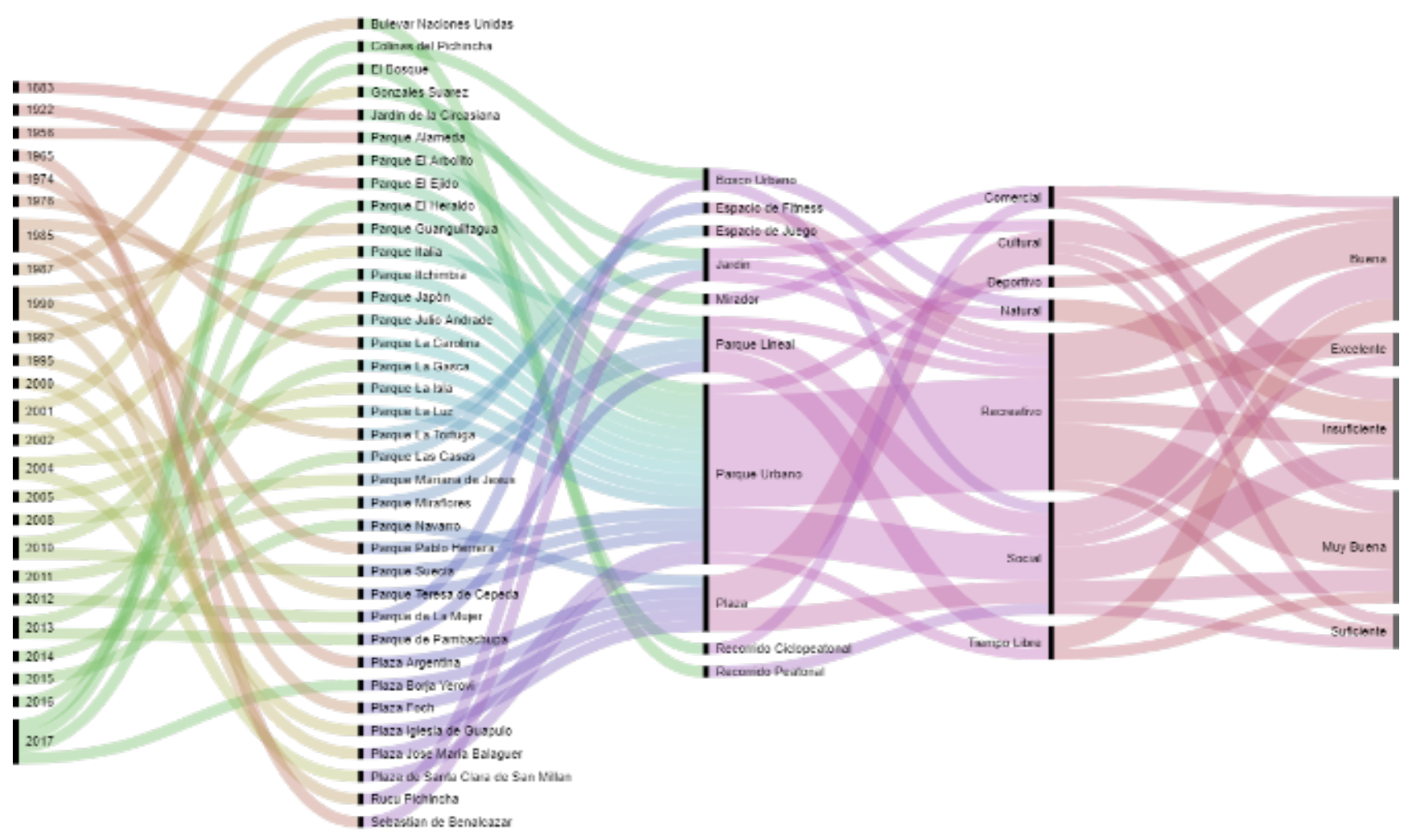


Aquel que aparece en cambio muy relevante es como, en ausencia de fuertes contenidos formales los espacios publicos se transformen en espacios aceptados donde la comunidad local interacciona y realiza actividades (figura 2).

Con condiciones meteorológicas favorables, grupos de personas pasan mucho de su tiempo de los días de fiesta sentados en círculo sobre el césped, cuentan historias, juegan, comen un bocadillo o un helado. Es decir, de un modo singular trasladan al espacio exterior las relaciones más estrechas del nivel familiar o de amistad, que se desarrollan generalmente en contextos cerrados.

La socióloga americana Jane Jacobs (1961) y el arquitecto Jan Gehl han tomado en consideración el espacio público occidental como espacio de relación de primer nivel, según un procedimiento de uso que pone a muchos usuarios a cierta distancia. Las relaciones que él puede establecer en el espacio externo solo son precursores con respecto a las relaciones más íntimas que pueden establecerse entre seres humanos; mientras el espacio público occidental, con buena paz de la idea de plaza como espacio de socialización, de solo hecho como un espacio de cruce (Desideri e Ilardi
1998).

La necesidad de llenar de contenidos formales y funcionales el espacio público desarrolla el papel de engagement para los usuarios, contestando a aquella aspiración de sociabilidad y a cohesión que la colectividad tiene un poco perdido, amparándose cada vez más en la esfera privada (Bauman 2012).

En ausencia de calles peatonales, destinadas para desarrollar la actividad lúdica de comprar, aunque los centros comerciales están empezando a imponerse como el Quicentro y el CCI, se observa el uso flexible de espacios públicos con la instalación a veces no completamente regular de tiendas informales en los parques públicos o puestos a lo largo de las calles colindantes.

La ciudad informal se auto-organiza por usos espontáneos de los espacios, siguiendo instancias básicas, contestando a necesidades contingentes de manera adaptativa Clemente (2005).

La smartcity occidental está absorta en crear lugares orientados al consumo de las calidades espectaculares, con un lenguaje reconocible que conduce a crear consumo visual y a empobrecer nuestra experiencia de vida urbana colectiva (Brain, 1977). Los mismos cascos antiguos, para huir del fenómeno del abandono, parecen destinados 
a convertirse en parques recreativos "temático" para turistas, a la insignia de la previsibilidad y el control (Sudjic, 1995).

En los espacios públicos del consumo de las capitales occidental, que se presentan como pedigreed landscapes (Rudofsky, 1964), dibujado según las concepciones estéticas dominantes del diseño urbano, induciendo comportamientos convencionales y preestablecidos, que les garantizan a las personas seguridad y protección.

El espacio urbano de Quito presenta condiciones imprevisibles debido a la flexibilidad de uso y a la espontaneidad de las configuraciones espaciales que los lugares pueden asumir con la presencia de las personas a las diferentes horas del día.

De otra parte, si la creación de espacios públicos orientados al consumo $\mathrm{y}$ de estructuras mono-funcionales especializadas y segregadas han creado una nueva geografía de la exclusión social (MacLeod 2002), los espacios públicos de Quito, que representa un caso de metrópolis "pobre", por el uso imprevisible y espontáneo, resultan ser espacios de verdadera socialización, en los que se da la posibilidad de cultivar relaciones de comunidad.

Los espacios públicos en Sudamérica son vividos de manera intensa por las personas, pues en estos lugares se reconocen, participan en la vida social de la ciudad y se sienten actores protagonistas de la vida urbana, no simples espectadores.

En estos espacios públicos el uso informal constituye la esencia de los lugares, que sin la presencia humana, también perderían su identidad social. El estudio de las prácticas informales puede ser una referencia útil para la realización de estrategias urbanas y prácticas de regeneración del espacio público, que toman como referencia la vida cotidiana de los espacios ordinarios (figura 5), como ocurre en Quito y en otros lugares más pobres del planeta, pueden volver a ser lugares de estar y compartir emociones.

\section{AGRADECIMIENTOS}

Los autores de este documento agradecen a la arquitecta paisajista Federica Perissi por el empeño y la dedicación en el análisis y elaboración gráfica de los datos recogidos.

Reconocen el gran trabajo desarrollado por los estudiantes del curso de "Introducción a la Ciudad y al Paisaje", del II semestre de 2017, de la Facultad de Arquitectura y Urbanismo de la UTE, para la recopilación de todas las fichas de los espacios públicos.

\section{REFERENCIAS BIBLIOGRÁFICAS}


Augé, M., y Rolland, D., (1993). Nonluoghi: introduzione a una antropologia della surmodernità. Milano: Elèuthera.

Bauman, Z., (2007). Homo consumens: Lo sciame inquieto dei consumatori e la miseria degli esclusi. Trento: Edizioni Erickson.

Bauman, Z. (2012). Modernità liquida. Roma: Gius. Laterza \& Figli Spa.

Borja J., Muxí Z. (2000). Espacio publico, ciudad y ciudadanía. Barcelona: Electa.

Carmona, M., Tiesdell, S., Heath, T., y Oc, T. (2003). Public Places, Urban Spaces: The Dimensions of Urban Design. Oxford: Architectural Press.

Chiesi, L. (2004). "Le inciviltà: degrado urbano e insicurezza". En R. Selmini (eds.). La sicurezza urbana. Milano: Franco Angeli, pp.129-140.

Clemente, M. (2005). Estetica delle periferie urbane: analisi semantica dei linguaggi dell'architettura spontanea (vol. 11). Roma: Officina.

Clemente, M., (2014). Strategia dei flussi pedonali nello spazio urbano aperto. En A. M. Ippolito (eds.), Spazi Urbani Aperti. Strumenti e metodi di analisi per una progettazione sostenibile. Franco Angeli, Milano.

Cobos, E. P., (1997). "La megalópolis neoliberal: gigantismo, fragmentación y exclusión". En Economía informa, No. 258.

Dematteis, G., y Rossignolo, C. (2006). "Città e reti di città in Europa". En "P. Bonavero, Dansero E., y Vanolo A., (eds.), Geografie dell'Unione europea. Torino: Utet.

Delgado, M., y Malet, D. (2011). El espacio público como ideología. Madrid: Los libros de la Catarata.

Desideri, P., e Ilardi, M. (1998). Attraversamenti. I nuovi territori dello spazio pubblico. Genova: Costa \& Nolan.
Gehl, J. (2014). Ciudades para la gente. Buenos Aires: Ed. Infinito.

Gehl, J. (2011). Life between buildings: using public space. Londres: Island Press.

Gehl, J. (1991). Vita in città. Spazio urbano e relazioni sociali. Rímini: Maggioli editore.

Gehl, J., y Gemzøe, L. (2004). Public spaces \& Public life. Copenhague: Danish Architectural Press.

Jacobs, J. (1961). Vita e morte delle grandi città. Saggio sulle metropoli americane. Torino: Piccola Biblioteca Einaudi.

Lefebvre, H. (1968). II diritto alia citta. Padua: Marsilio.

MacLeod, G., y Ward, K. (2002). "Spaces of utopia and dystopia: landscaping the contemporary city". En Geografiska Annaler: Series B, Human Geography, 84 (3-4), pp. 153-170.

Pea, A. (2016). "La rete ibrida degli spazi pubblici aperti". En F. Zagari, y F. Di Carlo (eds.), Il Paesaggio come Sfida. Il progetto. Melfi: Libria.

Pea, A. (2015). "The hybrid network of public open spaces", En A. Gospodini (eds.), Proceedings of the International Conference on Changing Cities II. Spatial, Design, Landscape \& SocioEconomic Dimensions, pp. 188-195. Tesaloniki: Grafima Publications.

Ratti, C. (2015). "Una cittadinanza sensibile per una città sensibile", Architetti Roma 113, pp. 26-27.

Reardon, T.; Timmer, C. P.; Barrett, C. B., y Berdegué, J. (2003). "The rise of supermarkets in Africa, Asia, and Latin America". En American journal of agricultural economics, 85(5), 11401146.

Rudofsky, B. (1964). Architecture Without Architects: a Short Introduction to Nonpedigreed Architecture. México DF: UNAM.

Sudjic, D. (1993). The 100 mile city. Nueva York: Harcourt Publishers Ltd. 


\section{Sitios electrónicos}

〈http://datos.quito.gob.ec/dashboards/10/dem ografià.

〈http://rawgraphs.io/〉. 